\title{
INVESTIGATIONS ON A PATIENT SUBJECT TO MYOCLONIC SEIZURES AFTER SENSORY STIMULATION
}

\author{
BY \\ G. D. DAWSON \\ From the Neurological Research Unit of the Medical Research Council, National Hospital, Queen Square.
}

(RECEIVED 29TH NOVEMBER, 1947)

\section{Introduction}

THE discharges which occur in the electroencephalogram (EEG) during myoclonic seizures have been described by Grinker, Serota, and Stein (1938), by Gibbs and Gibbs (1941), and by Jasper (1941a). None of these workers reported any effects of sensory stimulation on the course of the seizures or on the characters of the disturbances in the EEG. Two patients have been described (Dawson, 1946) in whom sensory stimulation under favourable conditions would provoke a myoclonic seizure. During the seizure which followed a stimulus such as a tap on a tendon, the discharges in the EEG seemed to be larger on the side of the head opposite to the limb stimulated. The evidence that this difference in the size of the discharge on the two sides of the head was related to the side of the body stimulated was not considered to be satisfactory. The purpose of this paper is to describe experiments carried out on a single subject in whom sensory stimuli provoke myoclonic seizures. These seizures are preceded by electrical disturbances which are detectable on the scalp and whose characters suggest that they are of cerebral origin. The disturbances appear largest on the side opposite to that stimulated; they appear near to the midline when the stimulus is applied to a leg and more laterally when it is applied to an arm. Studies have been made of the characters of these discharges and of the afferent systems concerned in their production.

\section{Material}

The patient, who was demonstrated at a clinical meeting (Carmichael, 1947), is a male, aged 42 years, with no family history of epilepsy or nervous disorder and no relevant past medical history. He attended the out-patient clinic for the first time in May, 1945, with the complaint that for eight months past when he put his weight on to his toes, or tried to climb a ladder, he had a feeling of uncertainty

c and later his feet began to shake. Also he had on several occasions fallen to the ground, but had been able to rise again immediately. There was no loss of consciousness or incontinence on these occasions. On one later occasion, just prior to admission, he had lost consciousness and bitten his tongue. Examination showed nothing but a suggestion of pyramidal dysfunction on the left side and he was admitted for fuller investigation in June, 1945.

After admission nothing abnormal was detected in the nervous system, in the cerebrospinal fluid, or in the skull by radiograph. An air encephalogram showed no abnormality. Dr. Hill reported that an EEG showed some abnormally slow rhythms in both parietal regions which were sometimes rather greater on the left than on the right side. These rhythms had a frequency of 4 to $7 \mathrm{c} / \mathrm{s}$. and they were augmented by overbreathing in the fasting state but not after the administration of $50 \mathrm{~g}$. of glucose by mouth. This finding was interpreted as being evidence against a diagnosis of idiopathic epilepsy, but there was no positive evidence of any localized abnormality or of an expanding lesion. Later during his stay in hospital the patient had two convulsive seizures in which the initial symptom was a drawing up of the legs. In these attacks consciousness was lost, he bit his tongue, and there was incontinence of urine. Treatment was started with phenytoin and phenobarbitone and he was discharged.

During the following eighteen months the patient became subject to severe myoclonic jerkings on which was superimposed, whenever he tried to walk, a bizarre rigidity in extension of the limbs and trunk. His rather cheerful indifference to his symptoms led to a diagnosis of hysteria.

In February, 1946, eighteen months after the onset of the illness, he was readmitted for further investigation; which again showed no evidence of any lesion that might be causing his symptoms. At 
this time the jerkings appeared locally, in which case they were almost invariably found in the muscles producing extension of the right big toe, or generally, affecting both legs and spreading to the arms, face, and diaphragm. During the less localized jerks the limbs became fixed for a period of half a second or less in an attitude between extension and flexion ; they did not show the great predominance of flexion that was characteristic of the two patients with myoclonus previously described (Dawson, 1946). The jerks occurred singly or in groups of diminishing severity separated by intervals of about a second or less. Examination of the reflexes was complicated by the fact that a tap on a tendon was usually followed by a generalized jerking whether the stimulus was applied to an arm or to a leg. Similar jerkings were produced by any sudden active or passive movement or by any stimulus to the patient such as a jar applied to the bed. Extensive sensory tests made by Dr. Norton showed that the only stimulus adequate for producing a myoclonic spasm was stretching of a muscle. Light touch, pinprick, heat and cold, or pressure, failed to cause any jerking so long as the stimulus was applied in a manner avoiding movement of a muscle. The threshold to stimulation was highest immediately after a previous jerk had occurred, and was then found to become progressively lower until finally a jerk would occur without any stimulus being applied, apparently spontaneously. The spontaneous jerkings occurred at intervals varying from a few minutes to a quarter of an hour, the frequency altering from day to day and remaining higher during periods of wakefulness than during sleep. On some occasions the jerkings seemed to be more frequent when the patient was starving, but raising the blood sugar did not prevent them and no abnormality was found in his tolerance to sugar. Increasing doses of phenytoin and phenobarbitone up to toxic levels made the jerkings worse, and when the drugs were withheld there was a considerable improvement in the patient's condition. Following this, amphetamine, $5 \mathrm{mg}$. three times a day, was given, and this resulted in a striking temporary improvement which was not, however, maintained.

The patient's condition remained stationary up to September, 1946, when he had a major convulsive seizure, the second since his readmission to hospital seven months before. This seizure began with a sensation of numbness which spread upwards from the left hand to the middle of the upper arm before consciousness was lost, This onset showed a change from that in earlier attacks, in which the initial symptoms were in the feet, especially the toes of the right foot. From that time the patient's condition has deteriorated slowly but steadily, with some remissions which have taken place particularly after major convulsions. The frequency of major seizures is now about three a month, with one free month in March, 1947, and one group of eleven seizures in twenty-four hours in May, 1947. Now he is quite unable to move without the occurrence of severe myoclonic jerkings which make articulation impossible. Between the more massive jerkings which occur almost continuously throughout the day and less frequently at night, there are coarse twitchings of groups of muscles all over the body and the plantar responses are now clearly extensor on both sides. The condition has been alleviated but not controlled by the administration of bromides. During the last five months the left hand and arm have become spastic and contracted in a slightly flexed posture, but are still involved in the jerkings. Apart from severe wasting the patient's general condition remains unchanged. No further laboratory tests have revealed any abnormality except the electrical investigations, the results of which are to be described. The cause of the condition remains obscure.

\section{Methods}

In these studies the differences of electric potential on the scalp were recorded by three different methods. For those experiments in which records over long periods were needed and when accuracy of recorded waveform was not of primary importance, ink-writing oscillographs were used. With the exception that the electrodes used were silver cups, coated with silver chloride and fixed to the scalp with collodion, the technique used was the same as that described previously for work on patients? with myoclonus (Dawson, 1946). When accuracy of the recorded waveform was important the inkwriters were replaced by cathode ray oscillographs and the record was made on continuously moving bromide paper. To indicate the time at which stimulation with a tendon hammer was applied it was arranged that the contact of the hammer with the skin discharged a small condenser through the subject, producing a brief deflection in the record. This deflection indicated a time slightly earlier than that at which the actual sensory stimulation occurred and for greater accuracy of timing an electric shock applied to peripheral nerve was used as the stimulus. The third method of recording used was that described for studies of the cerebral responses to sensory stimulation in healthy subjects (Dawson, 1947), where the methods for electrical stimulation have also been described. With this method of recording a number of records of the responses to stimulation are superimposed in such a way that the artefacts in the records due to the stimuli coincide. Any features of the responses occurring regularly after the stimuli tend to superimpose and are emphasized, whilst irregularities in the records unrelated to the stimuli become less prominent. The difficulties of timing the start of a response are thus greatly reduced. All records read from left to right and the values of the 

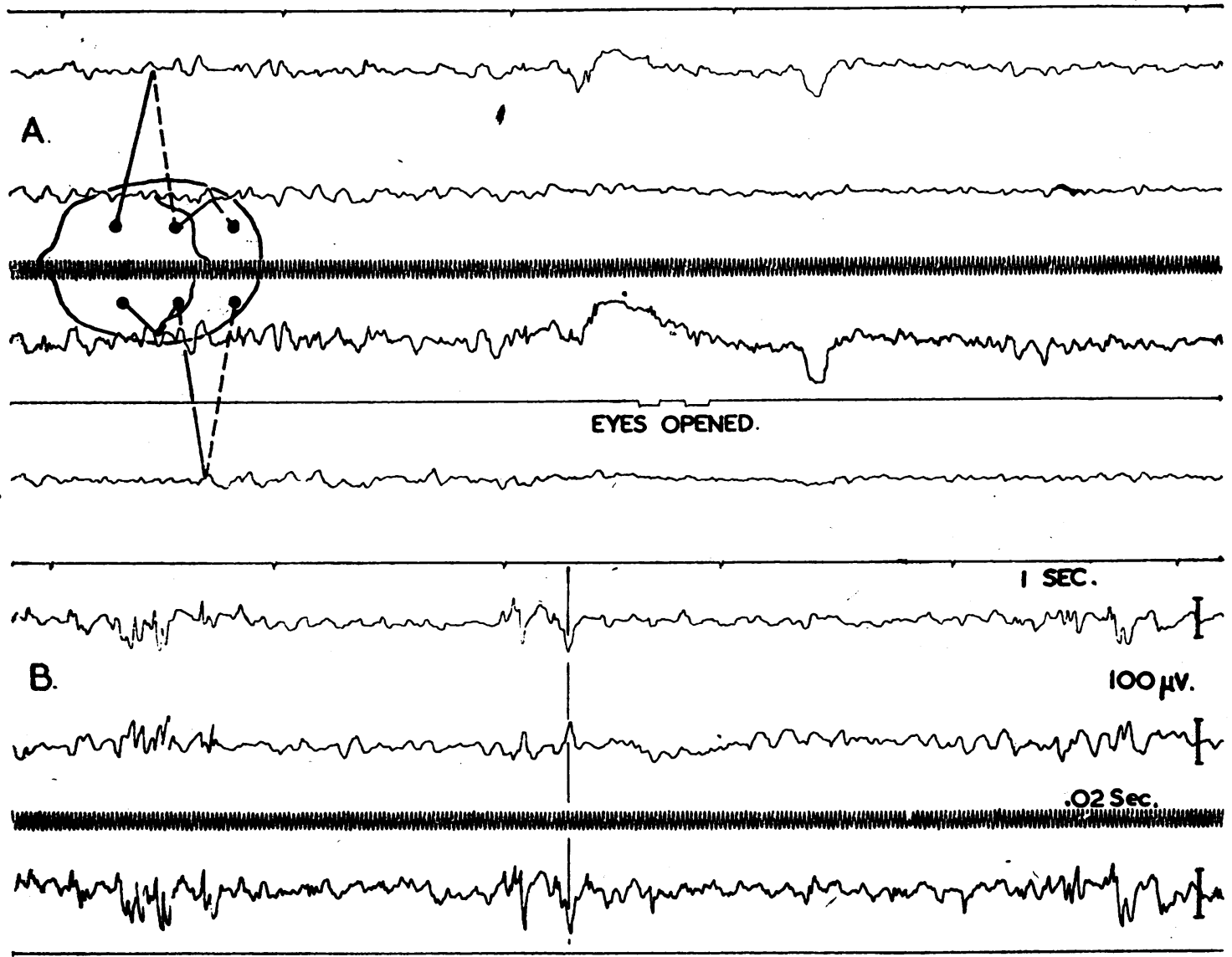

$$
\text { EVES SHUT. }
$$

FIc. 1.-Shows in A a section from the resting EEG of the patient between seizures. There are some rhythms at 8 to $10 \mathrm{c} / \mathrm{s}$. which are reduced on opening the eyes and there are some waves at 4 to $7 \mathrm{c} / \mathrm{s}$. on both sides. The record in B shows groups of spikes which were accompanied by myoclonic jerkings. The vertical co-ordinate through the middle group of spikes shows that the activity was greatest under the middle electrode on both sides. In this and all following records the continuous and broken lines to the electrodes in the head diagrams indicate that if the electrode connected to the broken line becomes positive with respect to that connected to the continuous line an upward deflection will occur in the record.

time scales and calibrations are indicated on the records or below the figures.

\section{Results}

The EEG Between Seizures.-The EEG of this patient taken at the time of his second admission to hospital, after the myoclonic jerkings had begun, shows the same features as those reported by Dr. Hill in earlier records. There is a rhythm of 8 to $10 \mathrm{c} / \mathrm{s}$. which is largest in the parieto-occipital regions and is reduced in size when the eyes are open, and there are some rhythms of low voltage with a frequency of 4 to $7 \mathrm{c} / \mathrm{s}$. which appear on both sides without any clear location. There are no rhythms of less than $4 \mathrm{c} / \mathrm{s}$. of significant size, and the record shows little change after overbreathing for three minutes. A section from the record before overbreathing is shown in Fig. 1A.

The EEG During Spontaneous Seizures.-In addition to the rhythms described above, the records show groups of spike-like discharges which occurred at intervals varying between a few seconds 
and fifteen minutes and were accompanied by brief myoclonic seizures. The record in Fig. 1B shows three groups of these spikes. Each spike has a period of 30 to 50 milliseconds and an amplitude of 50 to $150 \mu \mathrm{V}$. In the records from this subject the spikes that occurred spontaneously always appeared larger on the left than on the right side, though the difference was not sufficient to suggest an abnormality or lesion confined to the left side. The direction of the deflections in Fig. 1B shows that when the spikes occurred the activity was greatest under the middle electrode on each side and that in the first phase of the spike discharge the central regions became positive with respect to other regions in front and behind. After these brief seizures there was no extinction of or interruption in the cortical rhythms.

Using surface plate electrodes, records were made of the muscle action potentials during the seizures ; and such a record, from the quadriceps extensor muscles in the thighs, is shown, together with an EEG, in Fig. 2. The recording speed for this record was higher than for those in Fig. 1, and the waves in the EEG appear smoother and less like spikes than in the preceding records and they had a frequency during the seizure of thirty per second. The discharges in the EEG on the two sides appear to be synchronous and to have the same form within the limits of accuracy of this method of recording. The muscle action potentials also appear at the same time on the two sides, but they are considerably larger on the right side than on the left. This difference was observed constantly during spontaneous jerkings and agrees with the difference in the strengths of the jerkings that is observable clinically. The interval between the first of the spikes recorded in the EEG and the first of the muscle action potentials in this record is about 100 msets. The length of this delay apparently depends on the events immediately preceding the discharge. This is seen towards the end of the seizure where there is a gap of 120 msecs. in the spike discharge in the EEG. After the $120 \mathrm{msec}$. interval there is a pair of spikes, and the muscle action potentials associated with them follow after only 50 or 60 msecs. The apparent delay will also depend upon the amplification used in recording the muscle action potentials, since, if the potential differences do not reach their maximum size immediately, and it is clear from the records that they do not do so, they will be detected earlier with a higher amplification. Using high amplifications no sign of any action potentials significantly different from the resting activity in the muscles was found in less than 80 msecs. if the seizure did not follow within a few seconds of a previous outburst. The interval between the stimulus and the onset of the myoclonic jerking in the leg was not altered by more than 10 msecs. if the tendon tap was applied to the arm instead of to the leg itself. In the record

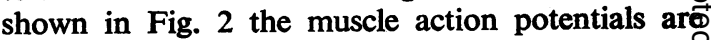
grouped with the same frequency as the waves io the EEG, but this is an exception, and in other records there is little sign of such a close relation in the rates of discharge. In none of the records from this subject during seizures do the muscle action potentials recruit in any regular manner, an $\frac{\phi}{7}$ their shape is very variable.
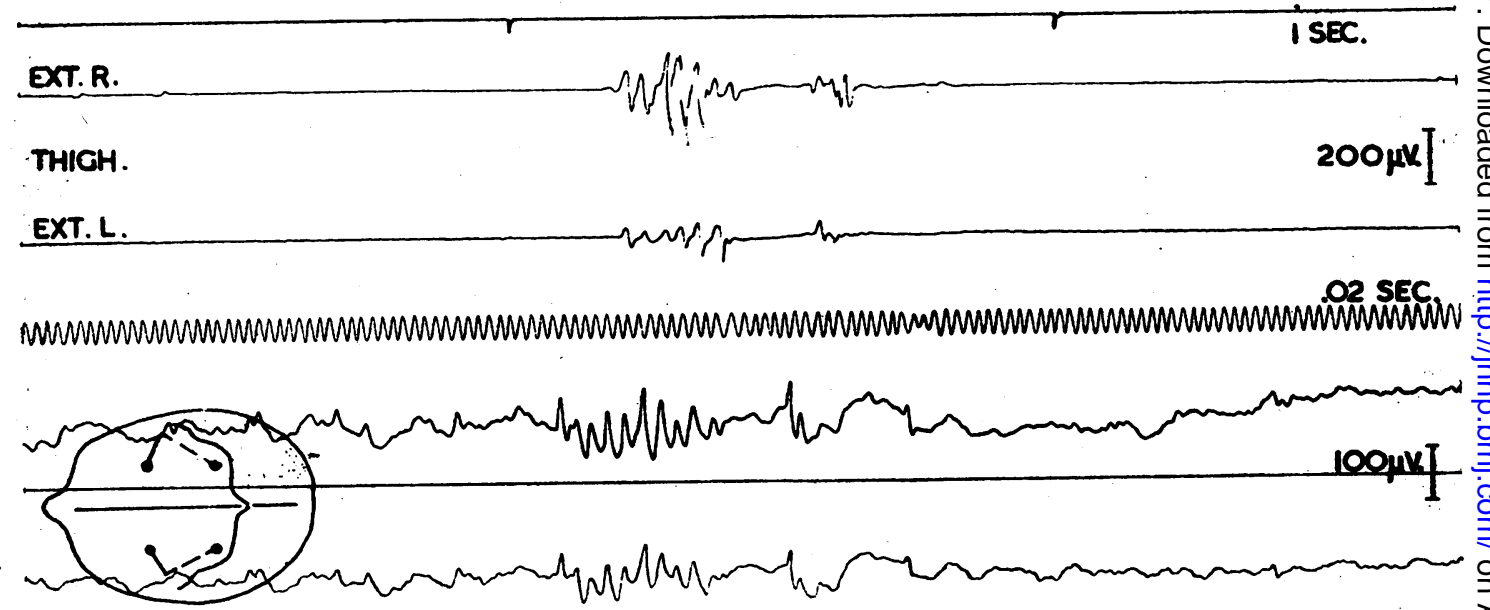

Fig. 2. -The two upper traces marked Ext. $R$ and Ext. $L$ record the muscle action potentials picked up from surface -plate electrodes on the extensor aspects of the right and left thighs. The two lower traces show the spike discharge in the EEG during a spontaneous myoclonic jerking. The spike discharge in the EEG has a frequency. $N$ of 30 per second and appears before the muscle action potentials. 


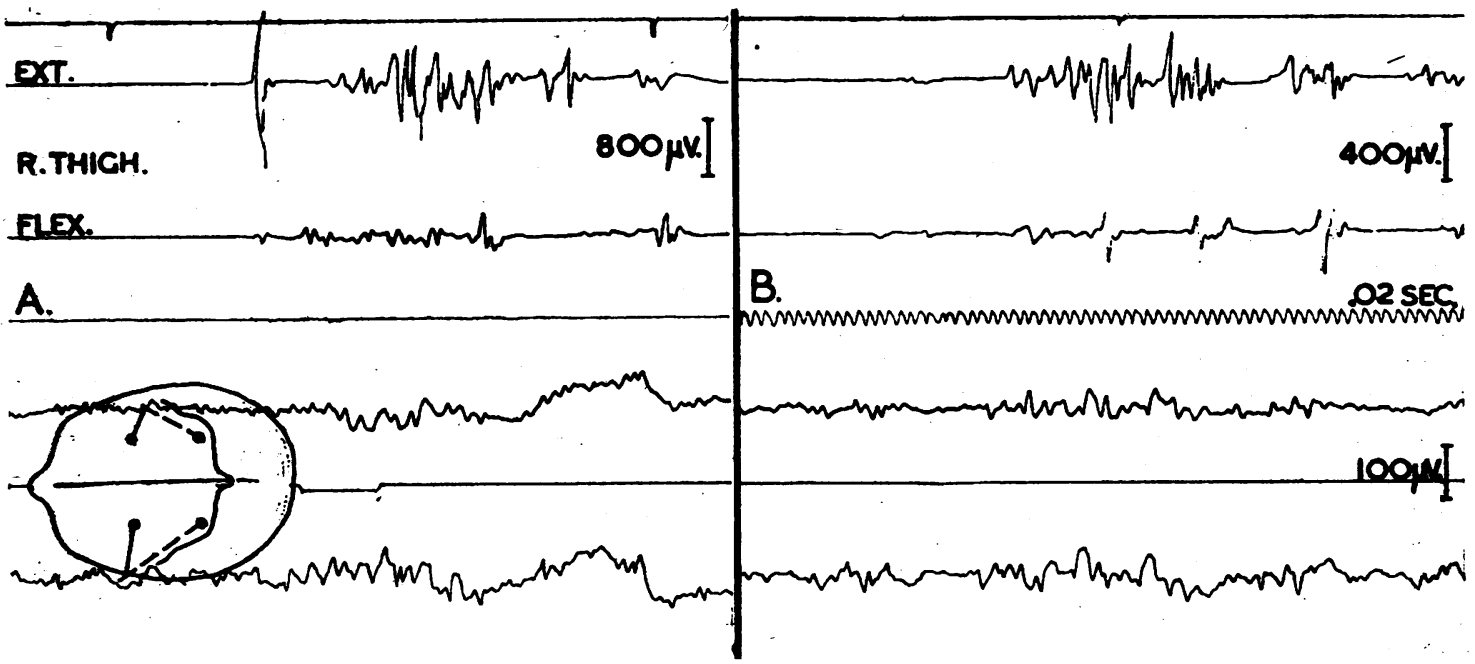

Fio. 3.-The records show the muscle action potentials from the extensor (Ext.) surface and flexor (Flex.) surface of the right thigh. In $A$ the patellar tendon was tapped and, following the knee-jerk action potential in the extensor muscles, a myoclonic jerk occurs, beginning first in the flexor muscles. B shows a spontaneous myoclonic jerk beginning at the same time in the two muscle groups.

Records During Provoked Seizures.-When a sensory stimulus is applied to this subject a generalized myoclonic seizure usually results. A record of the EEG and the muscle action potentials during such a seizure, provoked by a tap on the right patellar tendon, is shown in Fig. 3A. The muscle action potentials were recorded from the extensor and flexor surfaces of the right thigh. They show the action potential during the knee jerk from the extensor muscles followed by those during the myoclonic jerkings from both flexor and extensor groups. This and similar records show that the myoclonic jerkings following the tendon reflex begin first in the flexor muscles of the knee. This order of discharge is not, however, characteristic of this type of myoclonic seizure when it occurs spontaneously. It may be seen from the record in Fig. 3B, made from the same muscle groups during a spontaneous myoclonic jerking, that the action potentials begin either synchronously in the two muscle groups or rather earlier in the extensors. The later onset in the extensors in the record in Fig. $3 \mathrm{~A}$ is probably due to the period of inhibition that normally follows the tendon reflex. In these earlier experiments the location on the scalp of the area where the response to stimulation was greatest had not been worked out and the small size of the waves in the EEG in the records in Fig. 3 was probably due to the electrodes not being directly over this area of maximum activity.

The general level of relaxation of the subject was found to affect the strength and duration of the myoclonic jerkings greatly. When the subject was lying relaxed a tap applied to the patellar tendon produced a jerking that usually lasted little over a second. A similar stimulus applied one to two seconds after the subject had been told to clench both hands tightly resulted in jerking which was more violent than that in the relaxed state and which lasted for over five seconds. This facilitation of the jerkings when a voluntary effort coincided with a stimulus has been seen regularly, and no spontaneous variations of the same order of size occurred over short periods of time. The clinical examination of the tendon reflexes in this subject is difficult on account of the myoclonic jerkings that follow the reflex responses. In the record shown in Fig. 4A a tap was applied to the right patellar tendon and a large knee jerk action potential is followed by a spike discharge in the EEG and a myoclonic jerk. In Fig. 4B a stimulus applied to the left side produced a hardly detectable knee jerk action potential, but the subsequent myoclonic jerk was of the same size and has substantially the same form and duration as that which occurred when the stimulus was applied to the right side. This gave the appearance to clinical observation that the reflexes were brisker on the left than on the right side, the opposite to what was in fact the case.

In the records in Fig. 4, and in succeeding records where the stimulus was a tap with a tendon hammer, the black dots above the traces indicate the artefacts which show the time when the tendon hammer hit 
the skin. The spike discharge in the EEG in the records in Fig. 4 shows features which have been seen repeatedly in these discharges. Following the deflection produced by the hammer contact is a pair of waves in which the deflection is upward from the baseline. With the connexions to the amplifier used this indicates that the back electrode on each side became positive with respect to the front one. Following these two waves, which are separated by about 30 msecs., there is a slower downward deflection lasting for 100 msecs. on which is superimposed the spike discharge that is accompanied by the myoclonic jerking. During the slow downward deflection the back electrode on each side became negative with respect to the front one. In certain circumstances the first pair of waves was not followed by either the slow negative wave or by the rapid spike discharge. When this occurred there was no generalized myoclonic jerking, but a second brief jerk, which was confined to the stimulated limb, occurred after the main reflex response.

The form of the initial pair of waves and the succeeding events in the response to stimulation is more clearly shown in the records in Fig. 5, made with a cathode ray oscillograph. Records A and B in this figure show the complete series of events, the tap on the tendon indicated by the dot above the trace, the initial double positive wave, the slower negative wave, and the rapid "spike" discharge with a frequency of thirty per second. In both records the spike discharge slows down considerably just before it ceases. Records $C$ and $D$ in this figure show examples of initial discharges which occurred alone, and the form of the waves may be seen to be closely similar to that in records A and B, where they were followed by the other disturbances. The initial double wave was found to occur alone if a stimulus was applied soon after a previous discharge, either provoked or spontaneous. The effect of repeated stimulation about once per second is shown by the record in Fig. 6. The second section of the record follows the first without any interval. When first applied the stimulus produced the initial double positive wave, the slower negative change, and the train of waves accompanied by the myoclonic jerking. As the series of stimuli continued, the negative change and the spike discharge became smaller and the jerkings almost disappeared. It seems, therefore, that in the response in the EEG to a tap on a tendon two parts may be recognized. The first part consists of the double wave whose size and form do not seem to be greatly affected by rates of stimulation up to once per second. The second part consists of the slower wave and the spike discharge, both of which tend to disappear after several stimuli have been applied at one second intervals. To see if further differentiation between these parts of the discharge could be made, the effect was tried of compressing the carotid arteryD on one side. The pressure was maintained for upe to seventy-five seconds, and during this time the patellar tendon on the opposite side of the body was tapped at intervals. When this was done nos.

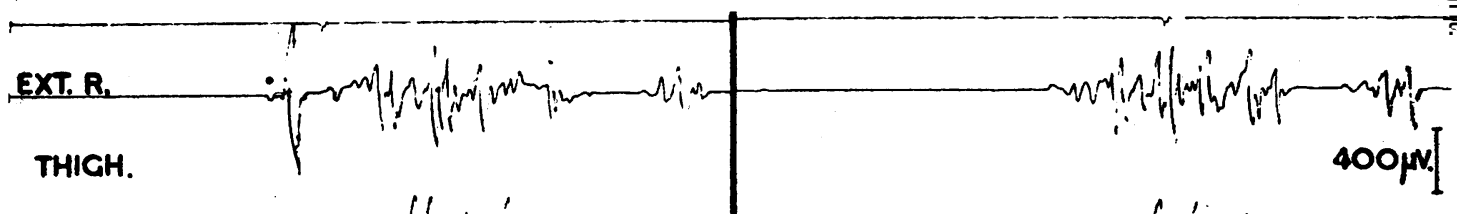
EXT.L.

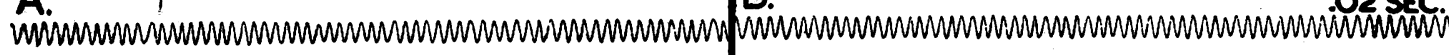

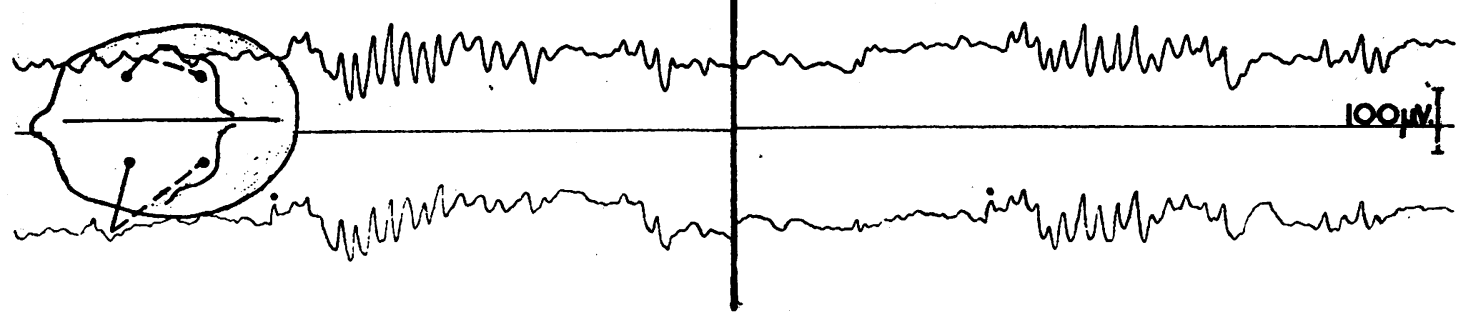

Fig. 4.-The records show the results of taps applied to A, the right, and $B$, the left, patellar tendon. Though the knee-jerk action potential following the tap on the left side is hardly perceptible, the following myoclonic jerk is as large as that which followed a much stronger knee jerk on the right side. (The black dot above the records indicates the artefact produced electrically by the contact of the tendon hammer with the skin.) 

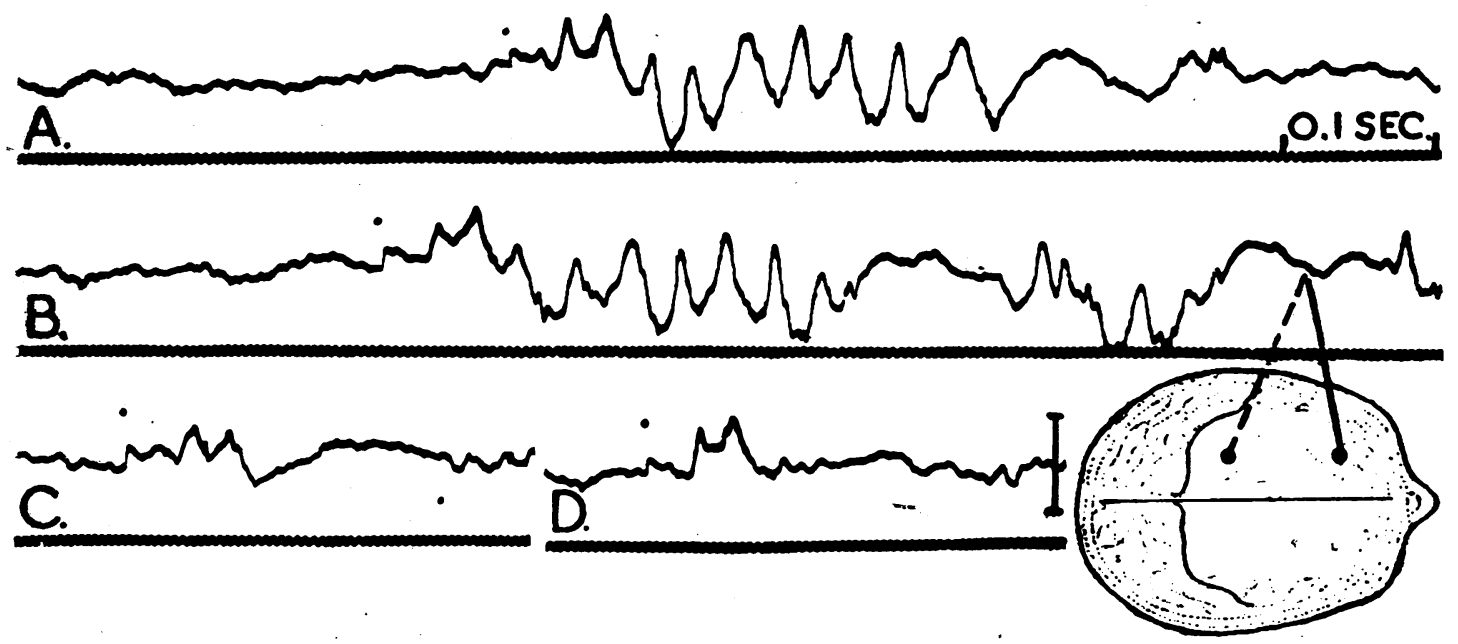

FIG. 5.-The records, made with a cathode ray oscillograph, show the form of the EEG discharge following a tap on a tendon. The black dots indicate the contact of the hammer with the skin. This is followed by a pair of waves during which the back electrode on the head became positive with respect to the front one. In $A$ and $B$ the back electrode then became negative and a train of spikes followed which was accompanied by a myoclonic jerk. In $C$ and $D$ no negative deflection occurred, and no train of spikes or myoclonic jerking followed. The calibration mark in D shows $100 \mu \mathrm{V}$.

difference was found in the ease with which jerkings or discharges in the EEG could be provoked and no alterations were found in the spontaneous cerebral discharges. This last observation suggests that although the subject stated that he had some sensation of light-headedness during the first period of compression, the degree of cerebral anæmia produced was not great and that therefore the value of the results is limited.

Attempts were made to measure the delay between the stimulus and the first most constant part of the response in the EEG more accurately than was possible in records such as those in Fig. 5. For this purpose the contact of the hammer with the skin of the subject was arranged to start the time sweep on the cathode ray tube and the sweep was photographed on stationary bromide paper. Using this methed it was found that the interval between tapping a patellar tendon and the first of the waves in the EEG varied between 30 and 35 msecs. Since part of this variation was probably due to the uncertainty of the delay between the time the hammer touched the skin and the stretching of the muscle, some method was sought of timing the actual stimulation more accurately. An electrical stimulus to a peripheral nerve, strong enough to be maximal for the motor fibres in the nerve, was found to produce closely similar discharges in the EEG and the same type of myoclonic jerkings. The only difference was that when stimulating the ulnar nerve at the wrist the delay between the stimulus and the myoclonic jerk in the arm was as long as 250 to 300 msecs., about 150 to 200 msecs. longer than that between a tap on the patellar tendon and the myoclonic jerk in the thigh muscles, or between a tap on the tendon of the biceps muscle in the arm and the following jerk in the anterior tibial muscles. Since no data are available on the delay between a tendon tap applied to the arm and the following myoclonic jerk in the arm itself, it is not possible to say how far this difference is due to the difference in the site of stimulation and how far it is due to the type of stimulus applied. Using electrical stimulation the characters of the first part of the response in the EEG were so constant that in later experiments attention was concentrated almost entirely on this initial part of the discharge. So far it has been assumed that the discharges in the EEG following sensory stimulation are of cerebral origin. Other sources of potential difference on the scalp likely to be encountered in experiments such as these are twitches of scalp muscle and, when an electrical stimulus is used, spread of current from the point of stimulation. Though these sources of artefact have not been excluded directly, the characters of the responses to be described suggest strongly that the responses are cerebral in origin and are not due to either of the other causes mentioned.

Distribution of Potentials.-With any one of the electrode positions used in these experiments no 
significant variation was found in the distribution of the potential differences on the scalp following stimulation, either during the periods up to two and a half hours covered by individual experiments or the period of a year covered by the series of experiments. Since the distribution of the response did not alter, it was possible to plot the potential gradients on the scalp by recording successively from a number of electrodes with only two recording systems. When the results obtained in this way were compared with those from experiments in which four recorders were used simultaneously no difference was found.

In Fig. 7 A, B, and $\mathrm{C}$ are shown records taken from four electrodes placed along the midline of the head, one over the vertex, one $6 \mathrm{~cm}$. behind this, and two others 6 and $12 \mathrm{~cm}$. in front of it. The record in $A$ shows the response to a single stimulus applied to the left lateral popliteal nerve at the head of the fibula. The point at which the response begins is difficult to define on account of the irregularity of the baseline due to spontaneous activity from brain and scalp muscle.

The records in $\mathrm{B}$ and $\mathrm{C}$ show superimposed the responses to fifty successive shocks, each maximal for motor fibres, applied at a rate of one per second to the same nerve at the same point as in record A. Record $\mathrm{C}$ was made after record $\mathrm{B}$, not simultaneously. The time scale for records B and C, which is shown below record $B$, was slightly slower than that used for A. The time between the stimuli and the responses does not vary by more than two milli-

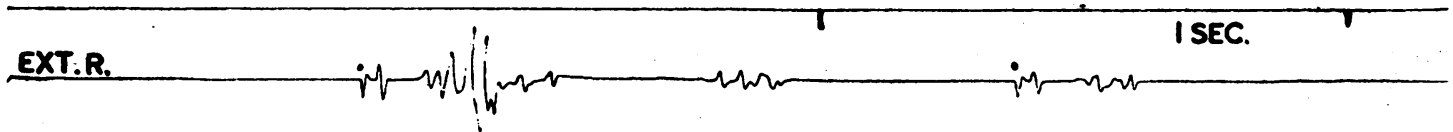

THIGH.

EXT.L.<smiles>CCCCCCC</smiles>

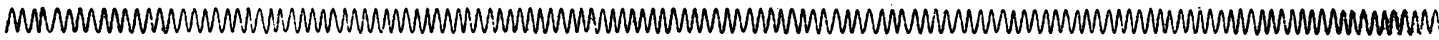
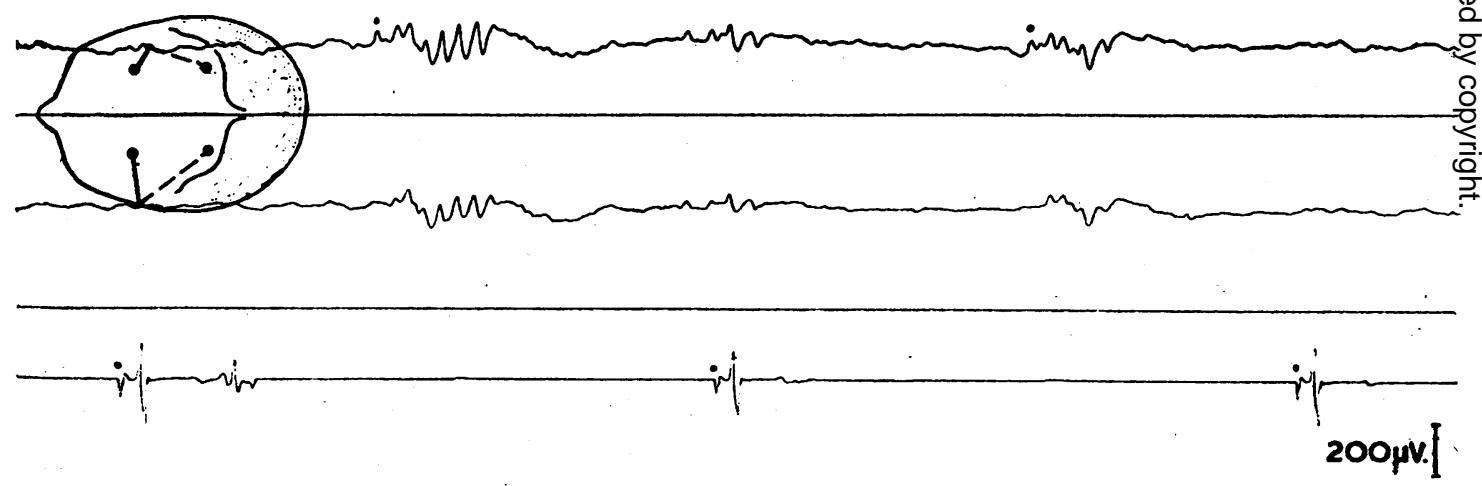

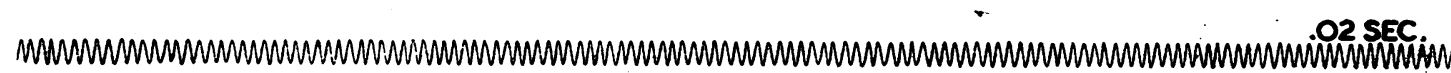

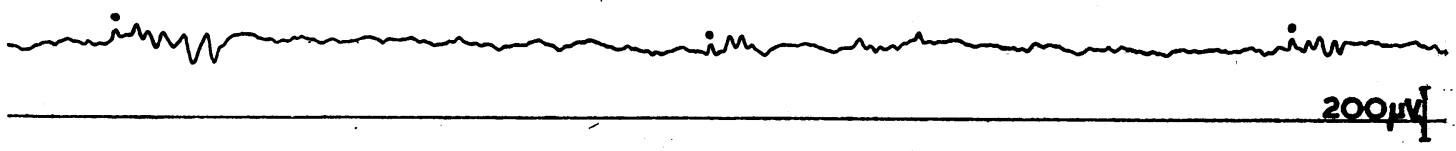

Fro. 6. -The records show the effects of a series of stimuli applied at intervals of one second to the right patellar: tendon. The initial pair of waves in the EEG, about 30 msecs. after the stimulus artefact, occurs in every case, but the spike discharge and the myoclonic jerking become smaller and almost disappear. 

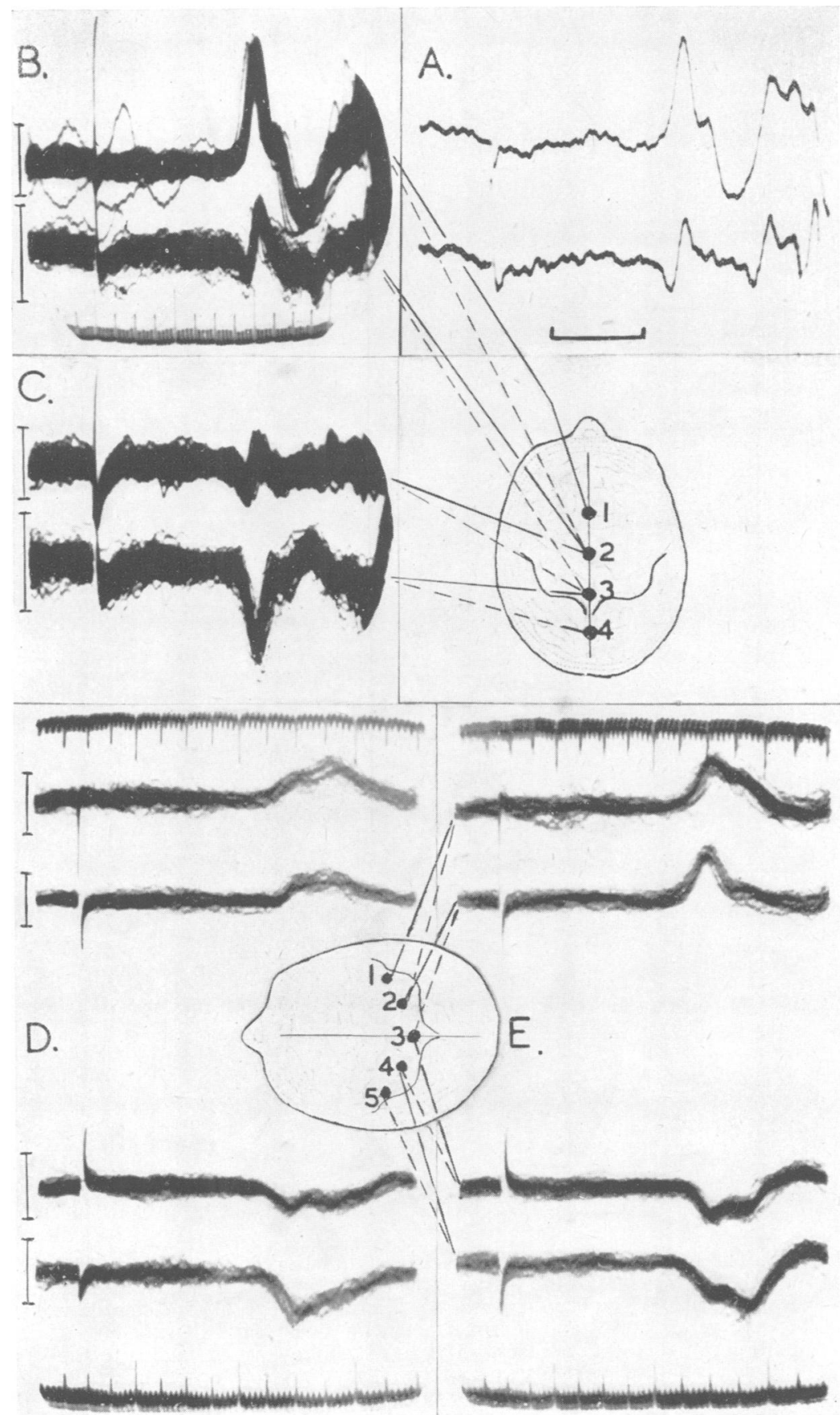

FIG. 7.-The records show the responses recorded from scalp following electrical stimulation of the lateral popliteal nerve at the head of the fibula. In $A$ is shown the response to a single stimulus; in $B, C, D$, and $E$ are shown superimposed the responses to 50 stimuli at one second intervals. Records B and C, made successively, show a maximum of activity, with the active area initially positive, between electrodes 2 and 3 . Records D and $\mathrm{E}$ show a maximum of activity near electrode 3 , on the midline. In $\mathrm{D}$ the stimulus was applied to the right side and the activity reaches a peak first in the records from the left side of the head. In E, where the stimulus was applied to the left side, the peak occurs first on the right side. The latency from the stimulus to the start of the response is between 33 and 36 msecs. The time mark below A shows 20 msecs. and the time scales in $B$ to $E$ show intervals of 1,5 , and 20 msecs. The calibration marks at the left hand ends of the records

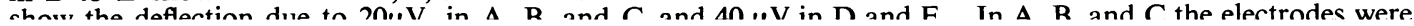




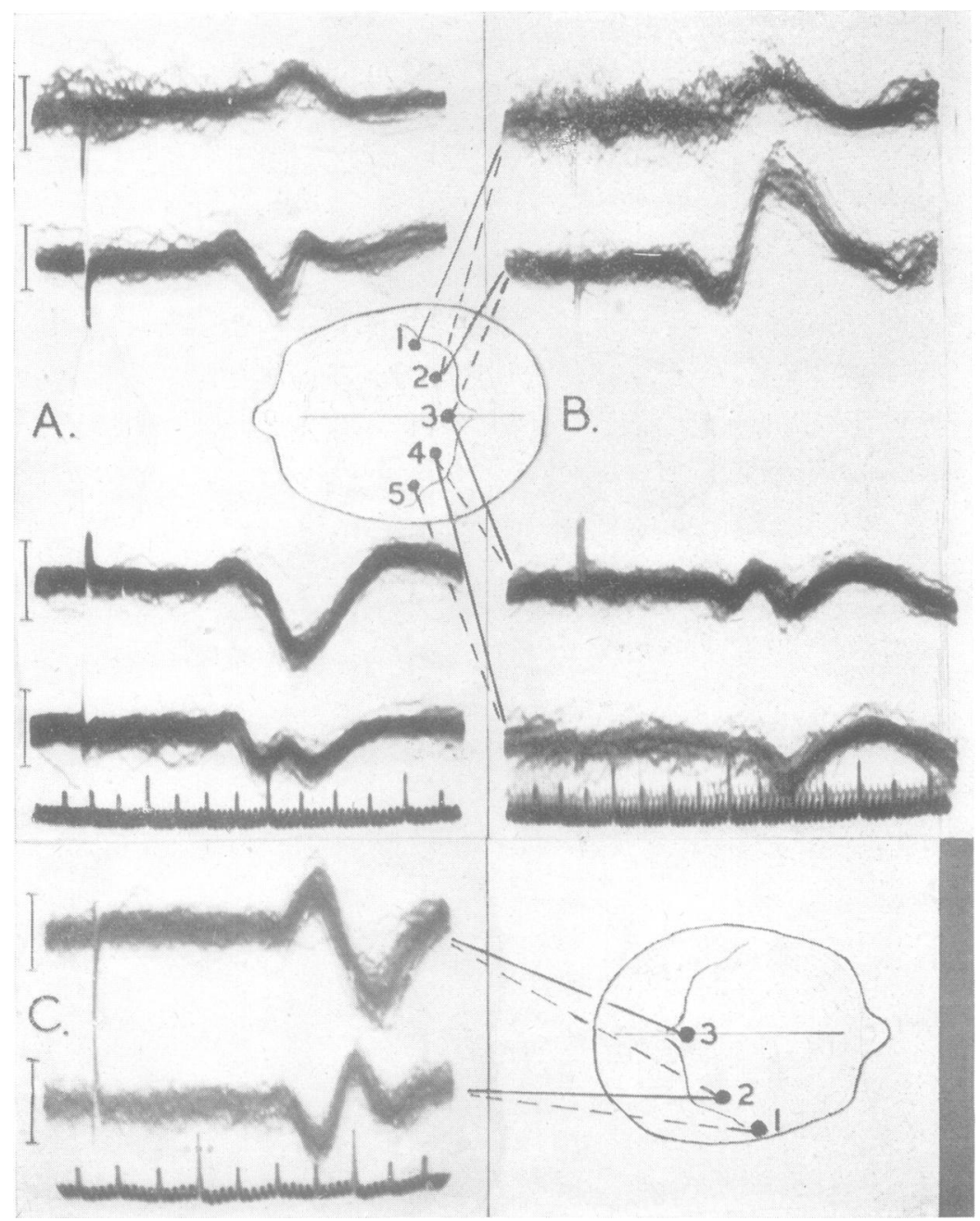

FIG. 8.-The records show the responses to stimulation of the right, $\mathrm{A}$, and the left, B, ulnar nerves at the wrists. The deflections appear largest in the leads from the side of the head opposite to the stimulus. In $\mathrm{C}$ the electrodes were on the vertex and 8 and $16 \mathrm{~cm}$. from it on a line parallel with the surface marking of the central sulcus. The activity following a stimulus to the left ulnar nerve at the wrist has a peak $8 \mathrm{~cm}$. from the vertex, nearer to electrode 2 than to either of the others. The time scales show intervals of 1,5 , and 20 msecs. The calibration marks for $\mathrm{A}$ and $\mathrm{B}$ indicate $40 \mu \mathrm{V}$, and for $\mathrm{C} 30 \mu \mathrm{V}$. 


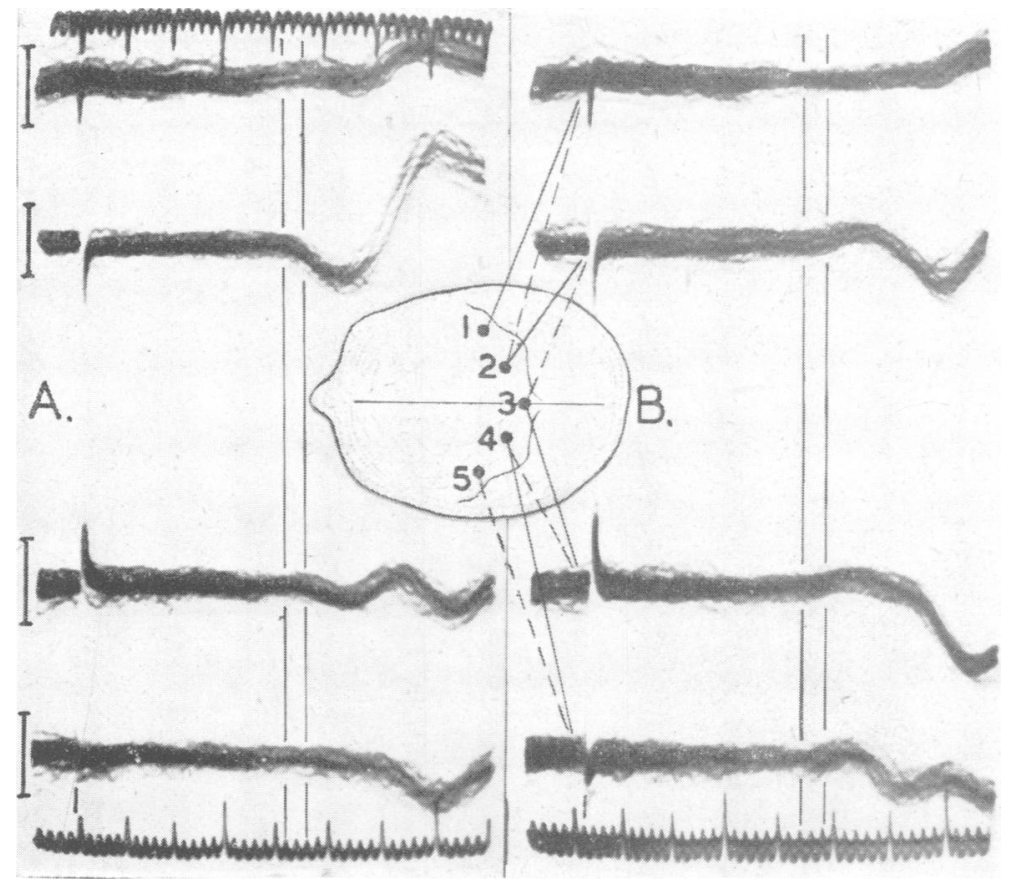

FIG. 9.-The records show the responses to stimuli to the left ulnar nerve at the wrist, A, and to the right ulnar nerve at the wrist, B, on a faster time scale than in Fig. $8 \mathrm{~A}$ and $\mathrm{B}$. The latency from the stimulus to the beginning of the response is 21 to 23 msecs., and the first activity that appears on the same side of the head as the stimulus, and which is not electrical spread from the opposite side, begins 7 to 8 msecs. later. The time scales show intervals of 1,5 , and 20 msecs., and the calibration marks $40 \mu \mathrm{V}$.
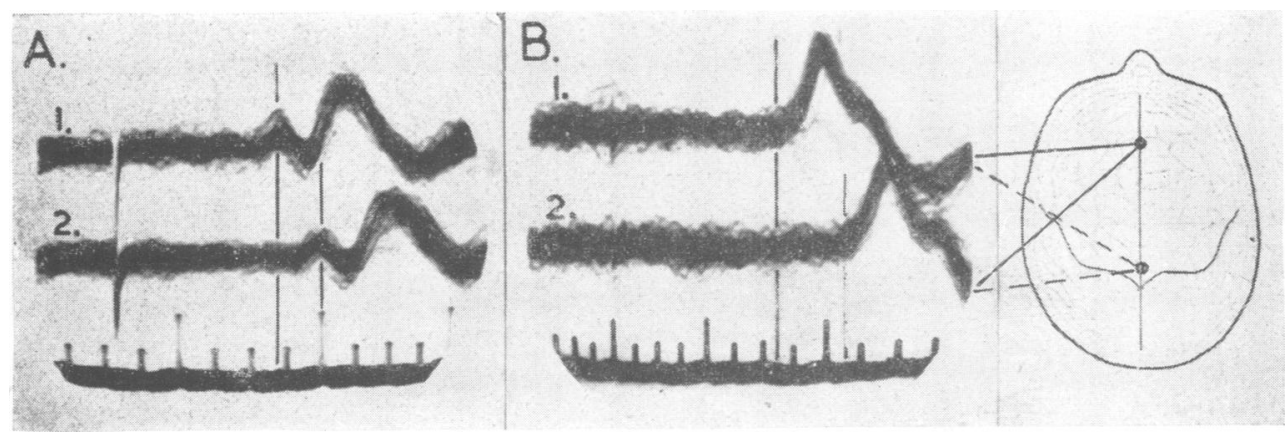

FIG. 10.-The records show in A the difference of 6 msecs. in the latency of the response following stimulation of the ulnar nerve at the elbow, trace 1 , and at the wrist, trace 2 . In $B$ is shown the difference of 10 to 11 msecs. in the latency of the response following stimulation of the lateral popliteal nerve in the popliteal fossa, trace 1 , and the posterior tibial nerve at the ankle, trace 2 . The time scales show 5 and 20 msecs. intervals. 


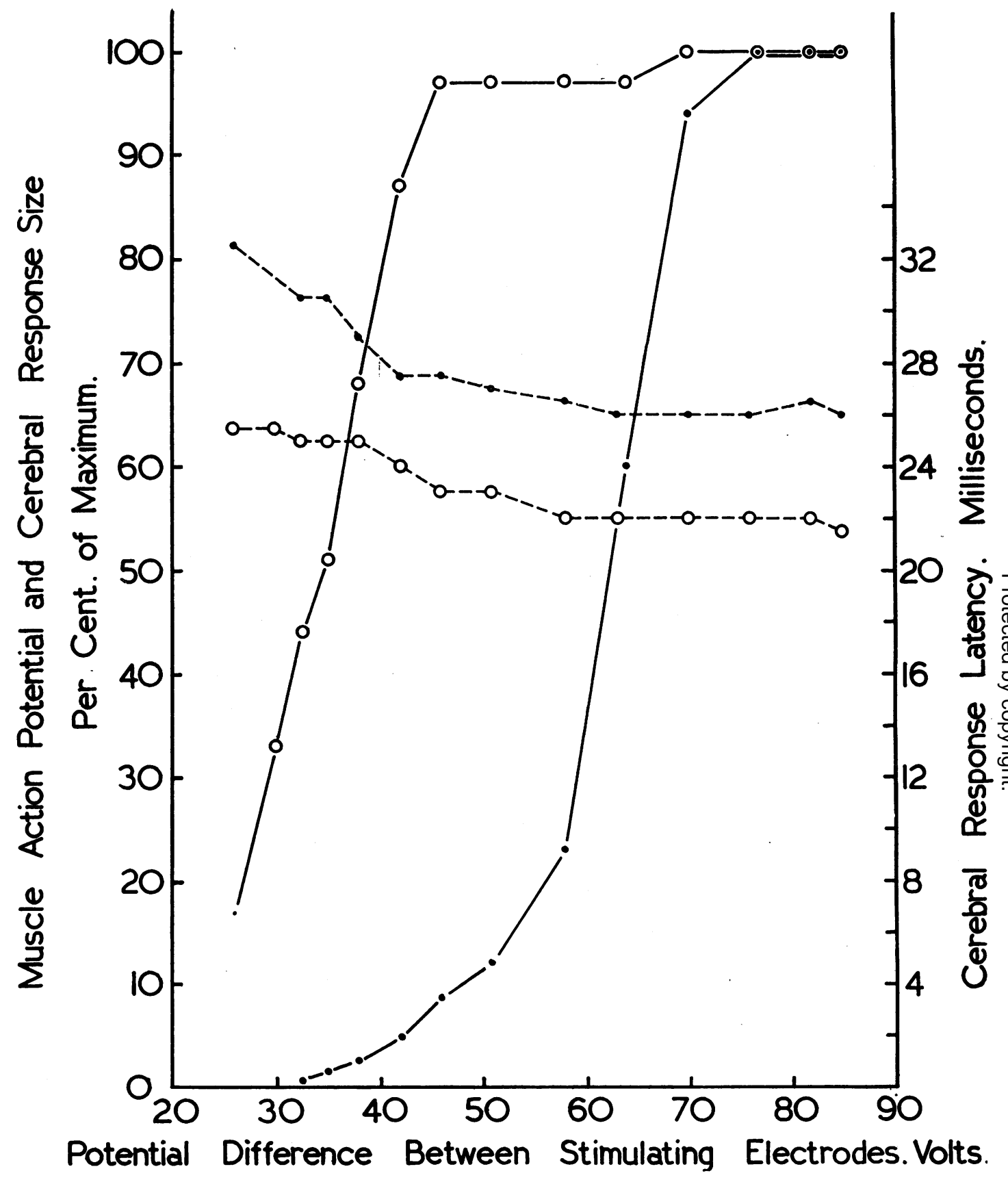

FIG. 11.- The graph shows the relation of the potential difference between the stimulating electrodes, with the cathode applied to the ulnar nerve at the wrist, and (1) the size from baseline to peak of the cerebral response (open circles and continuous line), (2) the size from baseline to peak of the action potentials from surface electrodes over the $\mathbf{M}$. abductor minimi digiti in the hand (dots and continuous line), (3) the latency from the stimulus to the start of the cerebral response (circles and broken line), and (4) the latency from the stimulus to the first positive peak of the cerebral response (dots and broken line). Each point is the mode of the results of fifty stimulations and probably closely approaches the mean value. 

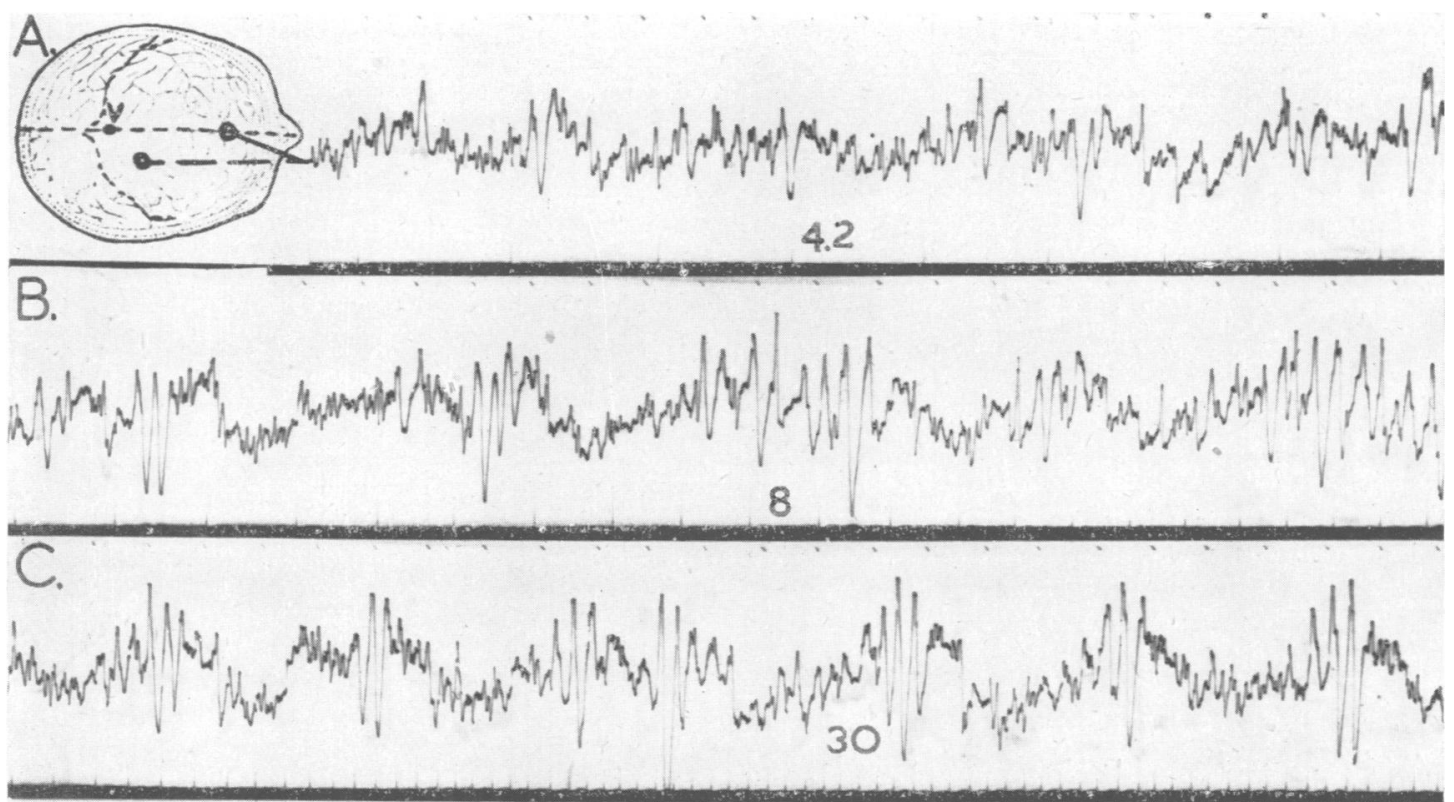

D.
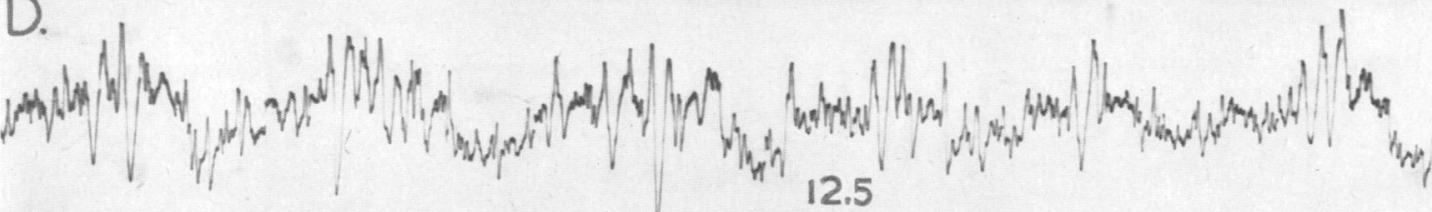

$E$

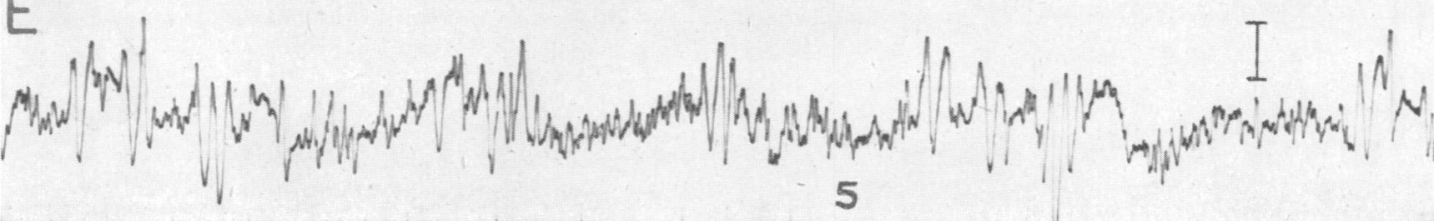

FIG. 12.-The record shows the EEG during stimulation of the left ulnar nerve at frequencies from 4 to 30 per second. The rate of stimulation is shown at the centre of each record and the spikes at the bottom of the records show the position of the stimuli. At a rate of 30 per second the stimuli provoke large bursts of oscillations at intervals of 0.3 to 0.4 of a second and with a frequency of 30 per second. These outbursts were accompanied by particularly violent myoclonic jerkings. The marks at the top of the records indicate intervals of $0 \cdot 1$ seconds and the calibration mark in $\mathrm{E}$ represents the deflection due to $50 \mu \mathrm{V}$.

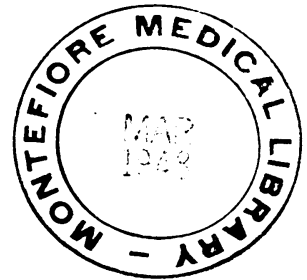



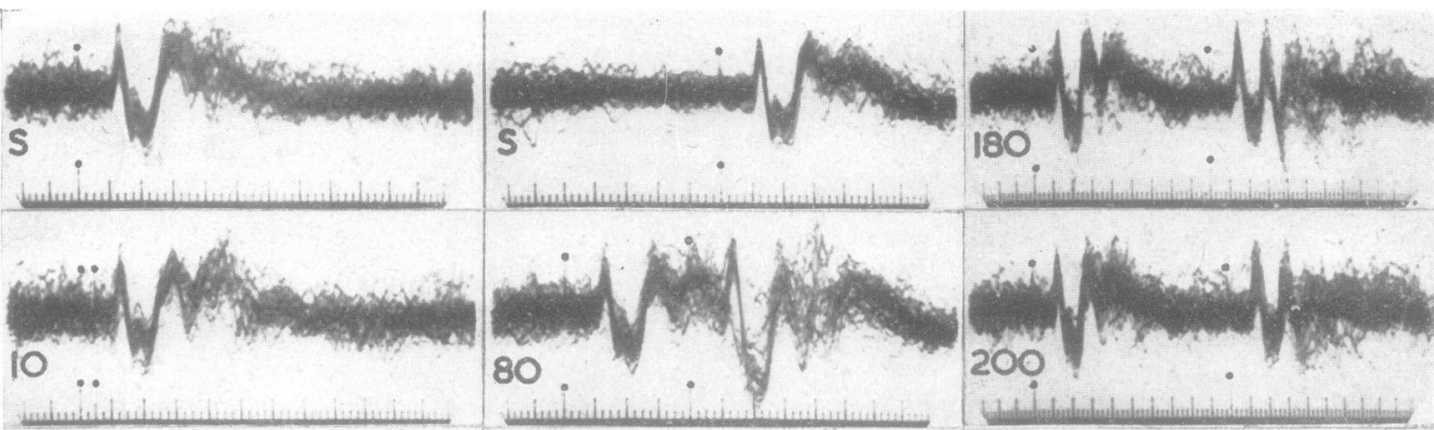

10
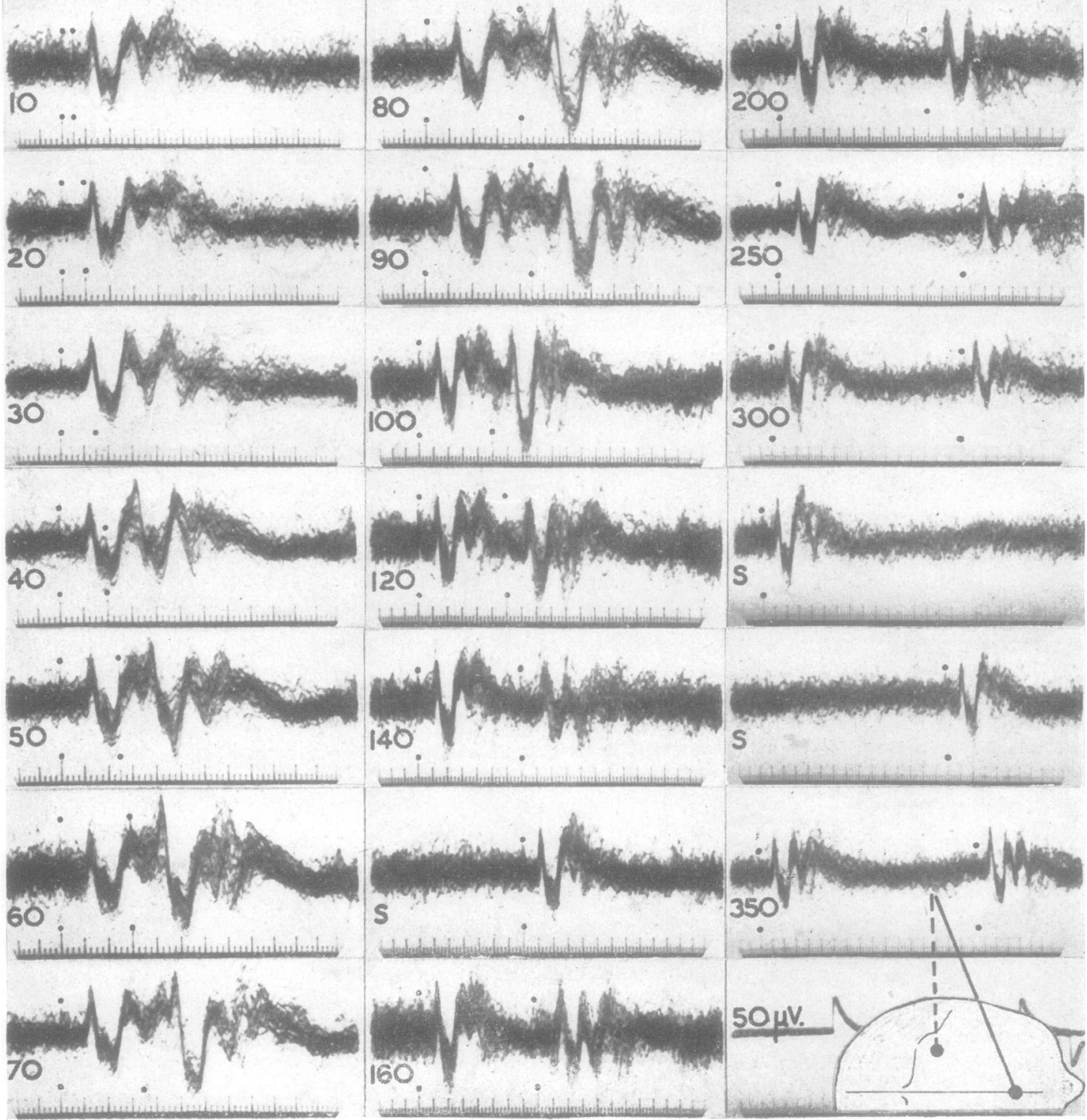

Fig. 13. - The records show the responses to a pair of shocks separated by intervals of from 10 to 350 msecs. $\mathrm{S}$ indicates single control stimuli, and in each record fifty single shocks, or pairs of shocks at the separation marked on the record, were applied at one second intervals. The black dots indicate the stimulus markers in the time scale and the stimulus artefacts in the cerebral response. The response to the second shock of the pair is depressed at a separation of the shocks of 10 to 30 msecs., it is facilitated from 60 to 100 msecs., depressed and delayed at 140 msecs., and almost returned to normal at 300 to 350 msers. The time scales indicated 5 and 20 msec. intervals. 
seconds in either direction in these records, and the form of the earlier phases of the response shows little variation. The sense of the deflections in the records in $\mathrm{B}$ and $\mathrm{C}$ shows that during the response the activity was maximal between electrode 3 , at the vertex, and electrode 2 , six centimetres in front of it. During the first phase of the response this area of maximum activity becomes positive with respect to other parts in front of and behind it.

The records shown in Fig. 7D and $\mathrm{E}$ were made with the electrodes arranged on lines parallel with and $3 \mathrm{~cm}$. in front of the surface markings of the central sulci. One electrode was placed on the midline and the others 4 and $8 \mathrm{~cm}$. from it on each side of the head. The records were made simultaneously from both sides of the head during stimulation of one or other lateral popliteal nerve at the head of the fibula. The stimulus was applied first to the right side, Fig. $7 \mathrm{D}$, and then to the left, Fig. 7E, and in both cases electrode 3, on the midline, became positive with respect to the others. The response also shows an asymmetry which is related to the side of the body to which the stimulus was applied. The first phase of the response consists of a positive wave that lasts for 15 to 20 msecs. on which are superimposed two briefer waves separated by 5 to 7 msecs. In the record in Fig. 7D, where the stimulus was applied to the right lateral popliteal nerve, the first of the two briefer waves is larger than the second one in the records from the left side of the head. In the records from the right side of the head the first of the brief waves is smaller than the second one. The difference is clearest in a comparison of the record from electrodes 4 and 5 and that from electrodes 1 and 2 . In Fig. 7E, when the left lateral popliteal nerve was stimulated, the opposite is the case, and in the records from the right side of the head the first of the two rapid waves is larger than the second. Thus the response to a stimulus applied to a leg appears as a potential change that is maximal about $3 \mathrm{~cm}$. in front of the vertex and near to the midline. Also it seems that the response reaches its peak sooner on the side opposite to that stimulated than on the same side as the stimulus.

The records in Fig. 8A and B, made with the same electrode positions as for those in Fig. $7 \mathrm{D}$ and $\mathrm{E}$, show the responses to stimulation of the right and left ulnar nerves at the wrists. The form of the response is different from that which follows stimulation of the lateral popliteal nerve, and the response is much larger on the side of the head opposite to the arm stimulated than on the same side. When the stimulus was applied to the right ulnar nerve, Fig. 8A, the greatest deflection appears in the lead from electrode 3 , on the midline, and electrode 4 , four centimetres lateral to it on the left side. The form of the deflections in the lead from electrodes 4 and 5 , on the left side, 4 and $8 \mathrm{~cm}$. from the midline, suggests that the disturbance was occurring between these electrodes at first nearer to electrode 5, when the deflections at the beginning of the response are in the same sense, and later moving medially to a point nearer to electrode 4 , four centimetres from the midline, when the deflections are in opposite sense. When the left ulnar nerve was stimulated, Fig. $8 \mathrm{~B}$, the greatest deflection appears in the record from electrodes 2 and 3 , on the right side of the head. In all these records electrodes 1 and 5 , which were $8 \mathrm{~cm}$. from the midline, were too close to it to allow the lateral extent of the area of maximal activity to be defined and all that may be said is that it was probably centred nearer to electrode 1 than to electrode 2, or nearer to electrode 5 than to electrode 4.

In Fig. $8 \mathrm{C}$ is shown a record taken from the right side of the head with electrodes over the same line parallel to the surface marking of the central sulcus but 8 and $16 \mathrm{~cm}$. from the midline. Here a stimulus to the left ulnar nerve at the wrist produces a deflection starting in opposite sense in the two traces, suggesting that the activity was at first maximal near to electrode 2, common to the two recording channels, and showing that the scalp in this area became positive with respect to other parts. In the later phase in which the active area has become relatively negative it has moved laterally so that it lies between electrode 2, and electrode 1, eight and sixteen centimetres from the midline respectively. The same distribution with a maximum about $8 \mathrm{~cm}$. from the midline has been found when stimulating the right ulnar nerve and recording from the left side of the head. Experiments with an anteroposterior arrangement of the electrodes showed that the area of greatest activity was in this instance, as when the leg was stimulated, centred about three $\mathrm{cm}$. in front of the surface marking of the central sulcus. This distribution of the responses to stimulation of the arm and leg suggests that they are of cerebral origin. There is no clear reason why the response, if it was a reflex twitch of scalp muscle, should appear near the vertex, where there is least muscle, after a stimulus to the leg, or why, when stimulating the arm, it should appear largest on the opposite side of the head $8 \mathrm{~cm}$. lateral to the midline.

The question arises as to what part of the potential differences picked up from electrodes on the same side of the head as the limb stimulated is due to cerebral activity on that side, and what part is due to spread of current from the much greater activity on the side of the head opposite to the limb stimulated. With the electrode arrangement shown in 
Fig. 9 cerebral activity of the same polarity in homologous areas on the two sides of the head, for example under electrodes 1 and 5 , would be recorded in the records from the two sides as deflections in opposite sense. If there were only one maximum of activity, for example under electrode 5 in Fig. 9, any current flow affecting the electrodes on the opposite side of the head would produce deflections in the same sense on the two sides. Using these criteria it may be suggested from the records in Fig. 9 that both effects are occurring. These records were made with the same electrode positions and the same stimuli as were used for the records shown in Fig. 8A and $\mathrm{B}$, but were made on a faster time scale.

In part A of Fig. 9 is shown the response to a stimulus applied to the left ulnar nerve. The deflection begins in the trace from electrodes 2 and 3 about two milliseconds before there is any deflection in the leads from the left side. The first deflection appearing from the left side is in the lead from electrodes 3 and 4 ; it is in the same sense as that from the right side and is therefore probably electrical spread of the activity from the right side. Deflections then appear in both traces from the left side of the head and they are in opposite sense to those from the right side of the head. These deflections might be due to either fresh cerebral activity starting on the left side in addition to that already present on the right side, or to a single peak of activity near to electrode 3 , on the midline.

The records in Fig. 8 suggested that the initial disturbance following stimulation of the left arm was centred between electrodes 1 and 2 . If the disturbance in the records in Fig. 9A originated in the same place and then moved to near electrode 3 , a point should occur in the record where the traces from electrodes 1 and 2 and from electrodes 2 and 3 would move in opposite sense with equal amplitudes. Since this does not occur it may be suggested that the activity in the initial part of the response remains centred lateral to electrode 2 and does not move to electrode 3 , on the midline. If the centre of activity, initially on the right side, does not move up to the midline, it seems that the deflection which appears in the record in Fig. 9 from electrodes 4 and 5 , on the left side of the head, the same side as that of the limb stimulated, indicates fresh cerebral activity on the left side of the head. This fresh activity on the left side starts about 7 to 8 msecs. after the activity first appears on the right side of the head, and in the initial deflection of the left sided activity electrode 5 became negative with respect to electrode 4.

In Fig. 9B when the right ulnar nerve was stimulated a similar series of events occurred but with the activity appearing last under electrode 1 . This suggests that here also there was cerebral activity on the same side of the head as the stimulus, appearing later than the activity on the side of the head opposite to the stimulated limb. In these records in Fig. 9 there is evidence of a limited spread of the active area in a medial direction. In the record in Fig. $8 \mathrm{C}$ there is evidence of a similarly restricted spread in a lateral direction, and in the records in Fig. 7B and $\mathrm{C}$ of a movement of the area of maximum activity in a backward direction from nearer electrode 2 to nearer electrode 3.

Further experiments with the electrodes separated by three centimetres along the midline of the head showed no evidence of movement of the active area over distances greater than three centimetres in an antero-posterior direction. It seems, then, that three forms of spread may be detected in these records. First there is the local spread for up to three centimetres or less around each area of maximum activity. Secondly there is the purely electrical conduction of currents from an area of relatively great activity to surrounding less active or inactive areas. Thirdly there is the appearance of activity on the same side of the head as the stimulus, up to 8 msecs. later than the primary activity on the side opposite to the limb stimulated and of opposite sign to this.

Afferent Pathways. - When a nerve was stimulated at the periphery the delay between the stimulus and the response in the EEG was found to be longer than when the same nerve was stimulated proximally. This difference in the delays has been taken as evidence that the response on the scalp is produced by a disturbance propagated in nerve and not by spread of the stimulating current, and it has been used to make an estimate of the conduction velocity in the nerve between the two points of stimulation. Measurements of the total delay have been made from the stimulus escape in the record to the start of the response and to the peak of the first positive wave. The measurements were made to the middle, or the darkest part, of the thick line produced by the superimposed records; they are therefore the mode of fifty responses and, although no experiments have been carried out to determine the distribution of the sweeps in the thick record, it seems likely that this is close to the mean position of the trace. Though the delay from the stimulus to the response varied little in any one experiment, it varied from one experiment to the next. That part of this variation may have been due to differences in the positions of the electrodes on the head is suggested by the difference between the delay from the stimulus to the start of the response in the record 
in Fig. 8c, and that in trace 2 in Fig. 10A. In both cases the stimulus was applied to the ulnar nerve at the wrist, but in Fig. $8 \mathrm{C}$, where one of the recording electrodes was near to the active area, the delay was $23 \pm 1$ msecs., whereas in Fig. $10 \mathrm{~A}$, where the recording electrodes were farther from the active area, the delay was $25 \pm 1$ msecs. The range of latency from the stimulus to the start of the response that was found for the different sites of stimulation was from $19 \pm 1$ msecs. at the elbow to $24 \pm 1$ msecs. at the wrist, $35 \pm 2$ msecs. at the head of the fibula, $32 \pm 2$ msecs. in the popliteal fossa, and $43 \pm 2$ msecs. at the ankle.

For measurements of conduction velocity the nerve was stimulated at two points and the response recorded from a fixed pair of electrodes on the head. Fig. 10A shows records made in this way with stimuli applied to the ulnar nerve at the elbow, trace 1, and at the wrist, trace 2. The difference in the delay to the start of the responses is 5 msecs. and that to the first peak, shown by the vertical lines on the record, is 6 msecs. The distance between the stimulating points was $28 \mathrm{~cm}$. giving conduction velocities of 56 and 47 metres per second respectively. Fig. 10B shows the result of a similar experiment where the stimuli were applied to the medial popliteal nerve in the popliteal fossa, trace 1 , and to the posterior tibial nerve at the ankle, trace 2. Here the difference in the delay from the stimulus to the first part of the rise in each response is about 11 msecs. The difference in the delays from the stimuli to the peaks of the responses is less, about 10 msecs. The distance between the two stimulating points was $46.5 \mathrm{~cm}$., giving conduction velocities of 42 to 43 metres per second and 46 to 47 metres per second according to the point on the response used for the measurement.

There are several probable sources of error in such estimates of conduction velocity. The measurement of the distance between the two stimulating points over the surface of the limb probably does not give the length of nerve between the two points to within better than one centimetre in thirty, though no figures relating to such a measurement in the limbs have been found. The exact point of stimulation is uncertain owing to the spread of the stimulating current in the tissues, and experiments were made to see what was the order of this spread. If stimulation is occurring at a point on the nerve away from the electrode there must be some point at which the spreading current is just strong enough to excite the nerve. Any weakening of the stimulus would be expected to bring this point nearer to the electrode, and to increase the actual distance over which the resulting impulses would have to be conducted and therefore also the delay between the stimulus and the response. In the graph in Fig. 11 the delay between the stimulus and the first part of the rise of the response has been plotted as a function of shock strength. There was a slight but probably not significant increase in the delay between the shock and the cerebral response as the shock strength was reduced from 84 to 82 volts. A further reduction from 82 to 65 volts caused no increase in the delay. Since a twenty per cent. decrease in the size of the shock did not produce an increase in the delay it seems likely that, with the shock strengths used in these experiments, spread of the stimulating current was not a major source of error. The measurements of differences in time may be made to better than plus or minus $1 \mathrm{msec}$. Although this is a small part of the total delay from stimulus to cerebral response, it is large in comparison with the differences of 5 to 6 msecs. in the delays from stimuli at the wrist and elbow, and probably it accounts for a great part of the errors.

A further source of error lies in the fact that when the nerve is stimulated peripherally fewer fibres will be excited than when it is stimulated proximally after any branches have joined it. If the excitation of fewer fibres in the nerve causes a slower rate of rise in the cerebral response this would apparently increase the delay following the peripheral stimulus, and make greater the interval between the response due to it and that due to the proximal stimulus. To examine this source of error, and to try to estimate the threshold to electrical stimulation of the afferent fibres concerned in the response, the ulnar nerve was stimulated at the wrist and the cerebral response was recorded from over the appropriate area of scalp. At the same time a record was made of the action potential in the M. abductor minimi digiti in the hand and of the potential difference between the stimulating electrodes. Fifty records were superimposed, and in Fig. 11 there are shown plotted as a function of the potential difference between the stimulating electrodes, the delay from the stimulus to the start and to the peak of the cerebral response, the size of the cerebral response from baseline to peak, and the size of the muscle action potential from baseline to peak. The sizes are plotted as a percentage of the maximal sizes of the responses, and the value for each point on the graphs is the mode of fifty records. When the stimulus fell below 60 volts the delay from the stimulus both to the start and to the peak of the cerebral response began to increase and a significant change of $1 \mathrm{msec}$. occurred before the size of the cerebral response had fallen significantly. Thus, although there is no reduction in the size of the response to the more 
peripheral stimulus in the records in Fig. 10A and B, there may still be a greater central delay than after the proximal stimulus, if the effects of stimulating a nerve trunk where it is smaller are similar to those of reducing the shock strength and stimulating fewer fibres in the nerve. The values of conduction velocity measured were between 42 and 56 metres per second, and the overall accuracy was probably about plus or minus thirty per cent.

The last source of error discussed seems likely to be large and it would give rise to lower values of conduction velocity than the true one. Measurements of the conduction velocity in the motor fibres in the same stretch of nerve in this subject and in healthy subjects where there was no question of central delay giving a low result have given figures of $45 \pm 10$ metres per second. If in fact the velocities obtained for the afferent fibres are low, this seems to suggest that they. might have a conduction velocity higher than that of the motor fibres in the same nerve and also a lower threshold.

In the graph in Fig. 11 it may be seen that when the strength of the stimulus to the ulnar nerve dropped below 75 volts the action potential from the $M$. abductor minimi digiti, recorded from surface electrodes, began to fall off in amplitude in the usual sigmoid manner and at a shock strength of 46 volts had fallen to less than one tenth of its maximum size. At this size of shock the cerebral response was not significantly reduced in size. This suggests two possibilities, first that the afferent fibres concerned have a lower threshold to electrical stimulation than the motor fibres in the same stretch of nerve, or secondly that so long as more than a certain number of fibres is stimulated there is an explosive cerebral response which tends to reach a maximum size. That the first is the more likely explanation is suggested by the fact that when the size of the cerebral response does begin to fall it does so progressively, in a similar manner to that of the motor response. If an explosive cerebral response were occurring it might be expected that the response would disappear suddenly when the number of fibres stimulated fell below a critical value. The points at which the muscle response and the cerebral response begin to fall correspond to 77 and 46 volts respectively. These figures give the relative thresholds for stimulation of the least sensitive fibres in each group and the values for the majority of the fibres in each group have a similar ratio. No information has been obtained about the most sensitive fibres as the cerebral response became smaller than the irregularities in the trace. At the last point, corresponding to a stimulus strength of 26 volts, it could be said that there was still a cerebral response present, although its size was not accurately measurable, whereas the muscle response had already become too small to measure, even with greatly increased amplification, above a stimulus strength of 30 volts.

To see if further information could be obtained about the afferent fibres concerned in the response to electrical stimulation the blood supply to a limb was cut off. A pneumatic cuff inflated to above the systolic blood pressure was applied to an upper arm in one experiment and to a thigh in another. After 25 to 35 minutes of ischæmia, when the conditions in the limb were changing rapidly, the interval that necessarily occurred between the examinations of sensory function and of the cerebral response to electrical stimuli made difficult correlation of the results of the two tests and the experiments were abandoned. In the two experiments made it seemed that the cerebral response to electrical stimulation of the nerve did not fail significantly before voluntary movement was lost in the muscles supplied by the nerve being stimulated. One interesting observation was made in the experiment where the cuff was applied to the right upper arm. After ten minutes of ischæmia it was noticed that the right foot was in a state of continuous slight clonic movement. This movement persisted untis between two and three minutes after the circulation had been restored. At the end of the experimento the subject volunteered the information that his arm? had felt for much of the time as though it had been bent up at the elbow and the fingers had beeno clenched. No record was available as to whether this sensation, which may have been the same aso that of the "pseudo-cramp" described by Lewis; Pickering, and Rothschild (1931), began at the same time as the twitching in the right foot, but it may be suggested that possibly the twitching was caused by the discharge of afferent impulses which was giving the false sense of position.

Experiments were made to find if any cerebral response could be produced by stimulation of a sensory nerve containing no afferent fibres from muscle. When the stimulus was applied to a small cutaneous nerve and made strong enough to be painful no cerebral response was found to follow. Stimulation of the sural nerve at the external malleolus provoked a cerebral response, but the strength of the shock was such that it was difficult to avoid spread to muscles from the cathode and stimulation of muscles under the anode. No useful information was obtained concerning latencies in this case as it was not certain that the response was due to stimulation of the sural nerve or at what level stimulation was occurring.

Stimulus Frequency.-In the experiments so far described the rate of stimulation was maintained 
at about once per second. This rate was chosen because the severe myoclonic jerkings disappeared after three or four stimuli and the first waves of the cerebral response did not seem to be altered in shape from those that followed a single stimulus. In some experiment's where faster rates of stimulation were used for short periods it seemed that unusually severe myoclonic jerkings occurred. Experiments were therefore made to investigate the effect on the response of the stimulus frequency. At first the method used was to stimulate a nerve at a rate increasing steadily from once a second to fifty a second, and to take a continuous EEG record. The effect of such a series of stimuli is shown in the record in Fig. 12. At a rate of stinulation of $\mathbf{4 \cdot 2}$ per second, Fig. 12A, it is difficult to pick out any response amongst the spontaneous activity from brain and scalp muscle in the record. As the stimulation rate increases oscillations with a period of a thirtieth of a second begin to appear in groups at intervals of half a second. At a stimulation rate of thirty per second, Fig. 12c, the myoclonic jerkings had become very violent and there are large groups of waves with a frequency of thirty per second separated by intervals of 350 to 400 msecs. This grouping tended to be maintained after the stimulation rate had been reduced to 12.5 per second, Fig. 12D, and is still visible with 5 stimuli per second. The critical frequency of stimulation at which the myoclonic jerkings became violent and at which the responses to the stimuli became clear in the record seemed to lie between 8 and 15 per second.

In another experiment, in which the rate of stimulation was changed more slowly in an attempt to find this critical frequency more accurately, the subject passed into a major convulsive seizure. Since such seizures were relatively rare the method was not used further and experiments were made using one conditioning stimulus followed at varying intervals by a test stimulus. The pair of shocks was applied once per second and their separation was varied between 10 and 350 msecs. At each interval fifty pairs of stimuli were applied and the records of the cerebral responses were superimposed; a series of records made in this way is shown in Fig. 13. The time scales show intervals of 5 and 20 msecs., and the black dots indicate the positions of the stimulus markers in the time scales and the positions of the stimulus artefacts in the records of the cerebral responses. The intervals between the stimuli are indicated at the left hand end of the records and an $\mathbf{S}$ indicates fifty single control stimuli. The size of the single stimulus necessary to produce a maximal cerebral response was found, and the first and second shocks in all the records were made twenty per cent. larger than this. The stimuli were applied to the right ulnar nerve at the wrist.

The responses to the second shock of a pair are reduced in size when the second stimulus. follows the first by between 10 and 30 msecs. At intervals of 40 and 50 msecs. the response to the second shock is superimposed on the end of the response to the first shock, and it appears to be the same size as or slightly larger than the first response. From 60 to 100 msecs. the second response is increased in size by 50 to 70 per cent., the greatest increase being apparently at 60 to 70 msecs. Between 120 and 160 msecs. the second response is depressed in size. This depression is greatest at about 140 msecs., where it may be seen by comparison with the record below, showing the responses to fifty single control shocks at one second intervals, that the delay between the stimulus and the response is significantly increased and is more variable when the response is depressed. The reduction in size is greatest in the second, positive, phase of the response which also becomes more oscillatory. The first phase of the response has apparently returned to normal at intervals of 180 msecs. and longer, but the more oscillatory nature of the later part of the response persists with an interval between the stimuli up to 350 msecs. It is interesting to note that the period of facilitation between 60 and 100 msecs. corresponds to the frequency of tetanic stimulation at which the myoclonic jerkings began to become very severe and at which the large cerebral discharges began to appear in the record in Fig. 12. At this interval between the stimuli the subject also began to complain that they were unpleasant in character. The interval of 350 to 400 msecs. between the groups of discharges at the faster rates of stimulation in Fig. 12 apparently corresponds to the period necessary for recovery after a previous discharge, as shown by the records in Fig. 13.

\section{Discussion}

The evidence that the discharges detectable on the scalp following sensory stimulation are of cerebral origin lies first in the delay between the stimulation and the response and secondly in the distribution of the responses. As the point at which the stimulus was applied was moved farther towards the periphery the latency of the response increased, and the amount of the increase was consistent with the cause of the disturbance on the scalp being propagated along the nerve stimulated. Such an increase in latency would not be expected if the electrical change on the scalp was due to any direct electrical conduction of the stimulating current to the head. The short latency of 19 msecs. between a stimulus 
applied at the elbow and the response on the head makes it unlikely that the response was a twitch of scalp muscle caused by the volley of impulses in the afferent fibres stimulated. That a twitch of scalp muscle was probably not the cause of the changes of potential detectable on the scalp is also suggested by the distribution of these potential differences which were maximal on the side opposite to that stimulated. The response to stimulation of a nerve in the leg was maximal near to the vertex, where the amount of muscle is least, and when stimulating a nerve in the arm it was maximal 6 to $8 \mathrm{~cm}$. lateral to the midline. This distribution suggests strongly that the potential changes detectable on the scalp were originating in the primary sensory cortex where the limb stimulated is represented. The only point against this is that in all the experiments the activity provoked by the stimuli was found to have a maximum 3 to $4 \mathrm{~cm}$. in front of the surface markings of the central sulcus. Though this difference is probably at the extreme limit of variation of the position of the central sulcus with respect to the surface markings, it may still be possible that these potential changes detectable on the scalp following sensory stimulation do represent cerebral activity from the region of the post-central sensory cortex.

The considerations discussed apply only to the first part of the response, and when it is followed by a wave of negativity at the active area, and a group of waves with a frequency of thirty per second accompanied by a generalized myoclonic jerking, it is difficult to define the distribution of these disturbances, though it seems that they spread more widely. The occurrence of such a clear local response associated with the stimulus, following as little as 19 msecs. after it when stimulating at the elbow, and also the relatively rapid rate of thirty per second of the later discharges, distinguishes this subject from those with myoclonic seizures previously described (Dawson, 1946). In these subjects, even during the times when sensory stimulation would provoke seizures, no similar local response was seen, and the rate of the later discharge lay between 8 and 14 per second. Whether or not this difference in the rates of discharge during the myoclonic seizures would account for the differences in the muscle action potentials in the two types of case is not clear. In the subject of the present experiments the muscle action potentials during the seizures were less simple than those in the cases described previously, where they were as brief and discrete as the action potentials during a reflex twitch from a tap on a tendon.

The first local response to stimulation in the subject of the present experiments bears in some ways a close resemblance to the responses which have been described in healthy subjects in similar conditions of stimulation and recording (Dawson, 1947). The latencies of the responses following stimulation of arm and leg correspond closely with those occurring in the healthy subjects and the distribution of the response is the same in the coronal plane, though in the healthy subjects the area of maximum activity in all cases where a response was found corresponded more closely with the surface markings of the central sulcus. No anatomical difference between the myoclonic subject and the healthy subjects, such as an atrophy of frontal cortex in the myoclonic subject, which might account for the difference was demonstrated radiologically. The polarity of the disturbances following stimulation in the present subject and in the healthy subjects previously described, where in the first phase of the response the active area became positive with respect to other parts, appears to be the same, though in no case was anything comparable to the negative change and the following spike discharge in the subject with myoclonus seen in the healthy subjects. The only major difference in the local responses in the myoclonic and healthy subjects is that in the myoclonic subject the potentiad difference on the scalp during the response was fivg to ten times as large as in the healthy subjects. IT⿱ the condition producing these large initial responses $z$ in the patient with myoclonus is a general corticaf one, it might be expected that the myoclonie jerkings would more often progress into a generals. ized tonic-clonic seizure, similar to that which occurred in the subjects with myoclonus previously $\vec{\varphi}$ described, but this rarely happens. The form of . the discharge following stimulation of the lateral popliteal nerve, shown in Fig. 7A, corresponds closely with that of the responses following electrical stimulation of peripheral nerve in the monkey, recorded by Marshall; Woolsey, and Bard (1941) from exposed cortex after recovery from ether anæsthesia. This suggests, like the correspondence in the time course and polarity of the response with that in healthy subjects, that the initial discharge in the myoclonic subject is abnormal only in size and not in other qualities.

Marshall and others suggest that the discharge they described is associated with the arrival of the afferent volley at the sensory cortex. This suggestion is supported by the work of Forbes and Morison (1939), who showed that a similar wave could be recorded from the white matter under cortex following electrical stimulation of a peripheral nerve, and by the work of Adrian (1941) who showed that after complete removal of the sensory cortex a wave could be recorded from the white 
matter leading up from the thalamus, following stimulation by touch, which was virtually identical with that which had been recorded from cortex before its removal. If in fact the initial wave in the response to stimulation in the myoclonic subject and in the healthy subjects is comparable with those described in animals by Forbes and Morison, by Adrian, and by Marshall and others, and if it is associated with arrival of the afferent volley at the cortex and not with any subsequent cortical activity, as is suggested by the short latency and the polarity of the first phase of the response, then the cause of the great difference in size between the responses in the healthy and the myoclonic subjects must be sought below the cortex, possibly at the level of thalamus. What light the changes in the size of the response to the second of a pair of shocks throw on the site of the facilitation that occurs at certain intervals between them is not clear, but it may be noted that the time of recovery after a discharge, 350 to $\mathbf{4 0 0}$ msecs., is closely similar to the period of the wave and spike discharges which appear synchronously on the two sides in the EEG during certain epileptic seizures. Jasper (1941 b) suggested that these discharges might be initiated from diencephalic structures, and more recently Fortuyn and Jasper (1947) have shown that a cortical discharge of wave and spike form may be elicited at a rate of three per second by stimulation of the dorso-medial nucleus of the thalamus in cats.

In clinical examination the only stimulus found adequate to provoke the myoclonic jerking was one involving stretching of a muscle. The tests of the threshold for electrical stimulation of peripheral nerve also suggested that the nerve fibres concerned in the response were more sensitive than the motor fibres in the nerve and had the same order of conduction velocity. The failure to find responses that were undoubtedly due to stimulation of sensory nerves containing no afferent fibres from muscle may have been due to the size of the shock used being insufficient to excite enough fibres. Bigger shocks became very painful and it was difficult to prevent stimulation of neighbouring muscles. If the fibres which could be stimulated to produce the cerebral response are in fact the large afferent fibres from muscle, the low order of conduction velocity in them obtained in these experiments, not higher than 60 to 80 metres per second, needs further consideration when it is compared with the figure of 116 metres per second described by Lloyd (1943). Figures of 45 metres per second have been obtained in healthy subjects for the conduction velocity in the motor fibres in the ulnar nerve and also for the afferent fibres in experiments on the reflex response to electrical stimulation of the type described by Hoffman (1922) It seems possible that the lower conduction velocity in the peripheral nerves in the limbs in man may in part at least be due to lower temperature, since Lloyd's. figures were obtained in the cat presumably at $37^{\circ}$ to $38^{\circ} \mathrm{C}$., though the temperature is not specified, and it is known that in man the temperature in an unclothed limb at rest may fall considerably below this figure. The low conduction velocity in the afferent systems is not necessarily inconsistent with the clinical observations that the adequate stimulus for producing the jerkings was that of muscle stretch.

\section{Summary}

1. A subject is described in whom sensory stimulation in the form of stretching a muscle or electrical stimulation of peripheral nerve provokes myoclonic seizures.

2. Electrical disturbances in the EEG preceding and during the seizures are described. The first part of the potential change is largest on the side of the head opposite to the site of the stimulus and $3 \mathrm{~cm}$. in front of the surface marking of the central sulcus. It is maximal near to the midline when stimulating the leg, and 6 to $8 \mathrm{~cm}$. lateral to it when stimulating the arm.

3. The latency of the response on the scalp becomes greater when the stimulus is applied nearer to the periphery ; it varies from 19 msecs. stimulating at the elbow to 43 msecs. when the stimulus is applied at the ankle.

4. The response to the second of two stimuli is depressed if the second stimulus is applied within 30 msecs. of the first. The response is facilitated if the interval between the stimuli is from 60 to 100 msecs., it is depressed again following this, maximally at 140 msecs., and has recovered almost completely at 300 to 350 msecs.

5. It is concluded that these responses to stimulation are cerebral in origin.

6. Attempts to define the characters of the afferent systems concerned are described.

7. The findings are compared with those in healthy subjects. The responses to stimulation in the subject with myoclonus have a similar latency, form and time course to those in healthy subjects but they are between five and ten times as large.

\section{The significance of the findings is discussed.}

The author would like to express his gratitude to Dr. E. A. Carmichael, who joined in many of the experiments, for his constant advice and encouragement.

\section{REFERENCES}

Adrian, E. D. (1941). J. Physiol., 100, 159.

Carmichael, E. A. (1947). Proc. roy. Soc. Med., 40, 553. 
Dawson, G. D. (1946). J. Neurol. Neurosurg. Psychiat., 9, 5.

(1947). Ibid., 10, 137.

Forbes, A., and Morison, B. R. (1939). J. Neurophysiol., 2, 112.

Fortuyn, J. D., and Jasper, H. H. (1947). Abstracts of Communications, p. 368, The 17th International Physiological Congress, Oxford.

Gibbs, F. A., and Gibbs, E. L. (1941). "Atlas of Electroencephalography." Cambridge, Massachusetts.

Grínker, R., Serota, H., and Stein, S. (1938). Arch. Neurol. Psychiat. Chicago, 40, 968.
Hoffman, P. (1922). “ Untersuchungen uber die Eigenreflexe Menschlicher Muskeln. Berlin. Julius Springer.

Jasper, H. H. (1941a). In " Epilepsy and Cerebral Localization." Penfield, W., and Erickson, T. C., p. 438. Thomas. Baltimore. (1941b). Ibid., p. 425.

Lewis, T., Pickering, G. W., and Rothschild, P. (1931). Heart, 16, 1.

Lloyd, D. P. C. (1943). J. Neurophysiol., 6, 317.

Marshall, W. H., Woolsey, C. N., and Bard, P. (1941). Ibid., 4, 1. 